\title{
Bronchiolitis in Rheumatoid Arthritis
}

\section{Mamasaidov AT*, Salieva RSH, Azhimamatova RM and Musaeva BS}

Osh State University, Osh, Kyrgyzstan

*Corresponding Author: Mamasaidov AT, Osh State University, Osh, Kyrgyzstan.
Received: June 13, 2021

Published: July 19, 2021

(C) All rights are reserved by Mamasaidov

AT., et al.

\begin{abstract}
Aim of the Study: This study aimed to evaluate the incidence of bronchiolitis in patients with rheumatoid arthritis (RA) and identify common risk factors and most clinical presentations of rheumatoid bronchiolitis.

Materials and Methodology: In this study, enrolled 156 patients with a confirmed diagnosis of RA. All studied patients underwent chest computed tomography (CT) scan conventional clinic and functional examination of the respiratory system and methods of detection of risk factors.

Results: According to the CT report signs of bronchiolitis were found in 46 (29,5\%) of patients with bronchiolitis. Clinical symptoms of bronchiolitis found just in $24(52,2 \%)$ of cases patients with CT scan signs of bronchiolitis. Rheumatoid bronchiolitis associated with smocking, male sex, early age debuted RA, and high level of DAS 28, rheumatoid factor (RF), Anti-cyclic citrullinated peptide antibody (ACCP), and spontaneous B lymphocyte activation.

Conclusion: Bronchiolitis (based on CT scan report) is a frequent extra-articular symptom of RA, and it is a common type of pathology of the respiratory system in this disease and is observed in almost $30 \%$ of patients with RA. The clinical presentation of the identified only $50 \%$ cases of radiologically confirmed signs of bronchiolitis and other remaining $50 \%$ case a subclinical (less symptomatic) variant of rheumatoid bronchiolitis is determined. The estimated risk factors of rheumatoid bronchiolitis are cigarette smoking, male gender, early debuted RA, and high level of DAS28, RF, ACCP and SBL.
\end{abstract}

Keywords: Rheumatoid Arthritis; Bronchiolitis; Computed Tomography; Clinical Signs of Bronchiolitis; Risk Factors of Bronchiolitis

\section{Introduction}

Rheumatoid arthritis (RA) - is a chronic autoimmune inflammatory rheumatic disease, clinically manifested progressive involvement distal small joints, and quite often reveals extra-articular manifestations including the respiratory system [1-3].

One of the type respiratory system involvement is bronchiolitis, that rarely diagnoses by standardized clinical and functional methods of examination $[4,5]$, however, quite often found on chest CT scan $[2,6-8]$.
In the development of the pulmonary disease (including bronchiolitis) in RA, as well as in the pathogenesis of RA upon the whole, a significant role plays a high level of SBL $[9,10]$.

The reported risk factors of rheumatoid bronchiolitis are cigarette smoking, male gender, early debuted RA, and high level of DAS28, RF, ACCP, and SBL [2,6,11].

\section{Aim of the Study}

The current study aimed to determine and evaluate the incidence of bronchiolitis in RA according to chest CT data, although, to clarify the risk of rheumatoid bronchiolitis. 


\section{Methodology}

Inclusion criteria

Total 156 patients were involved with reliable diagnosis RA, relevant criteria The American College of Rheumatology (ACR 1987) and European League Against Rheumatism (EULAR) Congress 2010.

\section{Exclusion criteria}

The studied group excluded patients with rheumatoid arthritis and chronic concomitant diseases of the respiratory system such as Asthma, COPD, Emphysema, and lung cancer, etc., although, patients who had long-term exposure to toxic chemicals for airways.

All enrolled patients with RA underwent a chest CT scan for the determining signs of bronchiolitis. Patients with confirmed signs of bronchiolitis were obtained general accepted clinical examination to identify clinical symptoms of bronchiolitis: 1) history taking for confirming the presence of cough, sputum production, and/or breathing difficulties; 2) lung auscultation for the detection existence of pathological lung sounds such as wheezes or crackles; 3 ) pulmonary function test (PFT) evaluating the forced expiratory flow in 1 second (FEV1) and forced vital capacity (FVC).

For all involved patients, the fact of smoking, the age of the patient, the age of onset RA, the duration of the disease, and the presence of rheumatoid nodules were assessed, the activity of the disease was assessed by the DAS28 index (Disease Activity Score), and the levels of RF, ACCP and spontaneous activation of B-lymphocytes (SBL) was determined. At the same time, the SBL index was determined by the method of quantitative cytofluorimetry according to the level of synthesis of intracellular Ig by peripheral B-lymphocytes $[9,12]$.

Statistical analysis of the obtained data was carried out using the Statistics version 17.0 program, including the generally accepted methods of parametric and nonparametric analysis with the calculation of the t-test. Differences were considered significant at $\mathrm{p}<0.05$.

\section{Results}

The majority of CT scan findings were direct (centrilobular micro-foci and structures of the "tree-in-bud" pattern) and indirect (areas of "mosaic perfusion" and the phenomenon of "air trap") CT signs of bronchiolitis [13], as well as thickening of the distal bronchial tubes, deformation of their lumen, and bronchiectasis.

At the same time, direct signs of bronchiolitis (centrilobular micro-foci and structures of the "tree-in-bud" type) were detected in 15 (9.6\%), indirect signs of bronchiolitis (areas of "mosaic perfusion", the phenomenon of "air trap") - in $13(8,3 \%)$, thickening of the walls of the distal bronchi - in 19 (12.2\%), deformation of their lumen - in 18 (11.5\%) and bronchiectasis - in 6 (3.9\%) patients with RA (Table 1).

In the same RA patient, from 1 (one) to 3 (three) of the above CT signs of bronchiolitis were detected, in general, CT signs of rheumatoid bronchiolitis were found in 46 (29.5\%) patients with RA (Table 1).

\begin{tabular}{|c|c|c|}
\hline \multirow{2}{*}{ CT scan patterns } & \multicolumn{2}{|c|}{ Rate } \\
\cline { 2 - 3 } & $\mathbf{n}$ & $\mathbf{\%}$ \\
\hline $\begin{array}{c}\text { Direct signs of bronchiolitis (centrilobular } \\
\text { microfoci and structures as "tree-in-bud") }\end{array}$ & 15 & 9,6 \\
\hline $\begin{array}{c}\text { Indirect signs of bronchiolitis (areas of "mo- } \\
\text { saic perfusion" and the phenomenon of "air } \\
\text { trap") }\end{array}$ & 13 & 8,3 \\
\hline Thickening of the distal bronchi walls & 19 & 12,2 \\
\hline Deformation of the lumen of the distal bronchi & 18 & 11,5 \\
\hline Bronchiectasis & 6 & 3,9 \\
\hline Total & 46 & 29,5 \\
\hline
\end{tabular}

Table 1: CT scan distribution of bronchiolitis in RA $(n=156)$.

The character and frequency of detection of clinical symptoms of rheumatoid bronchiolitis, determined using conventional clinical methods, are presented in table 2.

As table 2 shown, cough identified inpatients 22 (47,8\%), sputum production in 19 (41,3\%), dyspnea in 20 (43,5\%), wheezes and crackles were found in $8(17,4 \%)$ and declined value of FEV1 and PEF below $80 \%$ predicted in $17(37,0 \%)$ patient RA with CT signs of bronchiolitis.

Taking into account the fact that from 1 to 6 of the listed signs were observed in the same patient, in general, the clinical symptoms of bronchiolitis were found in 24 (52.2\%) RA patients with CT signs of the distal bronchi (Table 2). 


\begin{tabular}{|c|c|c|}
\hline \multirow{2}{*}{ Clinical presentations } & \multicolumn{2}{|c|}{ Incidence } \\
\cline { 2 - 3 } & $\mathbf{n}$ & $\mathbf{\%}$ \\
\hline Cough & 22 & 47,8 \\
\hline Sputum production & 19 & 41,3 \\
\hline Dyspnea & 20 & 43,5 \\
\hline Wheezes and crackles & 8 & 17,4 \\
\hline Declined FEV1 < 80\% predicted & 17 & 37,0 \\
\hline Declined PEF <80\% predicted & 17 & 37,0 \\
\hline Total & 24 & 52,2 \\
\hline
\end{tabular}

Table 2: Frequency of occurrence of clinical symptoms of bronchiolitis in RA patients with CT signs of this pathology ( $\mathrm{n}=$ 46).

The revealed studied risk factors of bronchiolitis in RA patients with and without CT signs of this pathology shown in table 3.

As follows in table 1, the fact of cigarette smoking identified in $34,8 \%$ of RA patients with CT sings bronchiolitis, which is significantly high $(\mathrm{p}<0.01)$ than in RA patients without CT signs of bronchiolitis (19.1\%). CT sings of bronchiolitis found in 37,5\% patients RA male gender, and $27,4 \%$ in female gender and in men, the symptoms of rheumatoid bronchiolitis were found significantly more often $(\mathrm{p}<0.05)$.

The average age of patients RA with CT signs of bronchiolitis was $53,7 \pm 2,62$, as well as in patients RA without CT signs - 51,8 \pm 2,51 , so, there were no significant differences ( $p>0.05$ ).

The average age of RA onset with CT signs of bronchiolitis was significantly less $(\mathrm{p}<0.05)$ than in patients without CT signs of bronchiolitis ( $38.9 \pm 2.47$ years and $46.1 \pm 2.32$ years, respectively).

The median duration of RA with and without CT signs of bronchiolitis were 9,4 $\pm 1,15$ years and 7,9 $\pm 1,01$ years accordingly and a reliable difference between them was not found ( $p>0,05)$.

Rheumatoid nodules were found in $37,0 \%$ of patients with CT signs of bronchiolitis, it was significantly high $(\mathrm{p}<0,05)$, then patients without CT signs of bronchiolitis $(19,1 \%)$.

The DAS28 in patients with CT signs of bronchiolitis was significantly higher than $(\mathrm{p}<0,05)$, in patients with no CT, signs of bronchiolitis $(6,2 \pm 0,65$ score and 4,5 $\pm 0,48$ score accordingly).

RF and ACCP were found in $84,8 \%$ и $80,4 \%$ patients with CT signs of bronchiolitis, which was significantly higher $(p<0,05)$, than in patients without CT signs of bronchiolitis $(72,7 \%$ and 70 , $\%$ accordingly).
The level of SBL in the group positive CT sings of bronchiolitis has been shown significantly higher value $(p<0,05)$, then in negative CT sings of bronchiolitis comparatively $(149,1 \pm 3,11$ standard units and 137,3 $\pm 3,25$ standard units accordingly).

\begin{tabular}{|c|c|c|c|}
\hline Risk factors & $\begin{array}{c}\text { RA patients } \\
\text { identified CT } \\
\text { sings of Bron- } \\
\text { chiolitis (n = 46) }\end{array}$ & $\begin{array}{c}\text { RA patients } \\
\text { without } \\
\text { CT sings of } \\
\text { bronchiolitis } \\
\text { (n = 110) }\end{array}$ & p \\
\hline Smocking, n (\%) & $16(34,8 \%)$ & $19(19,1 \%)$ & $<0,01$ \\
\hline Male/female, n (\%) & $\begin{array}{c}12(37,5 \%) / 34 \\
(27,4 \%)\end{array}$ & $\begin{array}{c}20(62,5 \%) / \\
90(72,6 \%)\end{array}$ & $<0,05$ \\
\hline Age (year), M \pm m & $53,7 \pm 2,62$ & $51,8 \pm 2,51$ & $>0,05$ \\
\hline $\begin{array}{c}\text { Age of onset RA } \\
\text { (years), M } \pm \text { m }\end{array}$ & $38,9 \pm 2,47$ & $46,1 \pm 2,32$ & $<0,05$ \\
\hline $\begin{array}{c}\text { Duration (years), } \\
\text { M } \pm \text { m }\end{array}$ & $9,4 \pm 1,15$ & $7,9 \pm 1,01$ & $>0,05$ \\
\hline Rheumatoid nod- & $17(37,0 \%)$ & $19(19,1 \%)$ & $<0,05$ \\
\hline ules, n (\%) & $6,2 \pm 0,65$ & $4,5 \pm 0,48$ & $<0,05$ \\
\hline $\begin{array}{c}\text { DAS28 (score), M } \\
\pm \text { m }\end{array}$ & $39(84,8 \%)$ & $80(72,7 \%)$ & $<0,05$ \\
\hline RF+, n (\%) & $37(80,4 \%)$ & $77(70,0 \%)$ & $<0,05$ \\
\hline ACCP+, n (\%) & $149,1 \pm 3,11$ & $137,3 \pm 3,25$ & $<0,05$ \\
\hline $\begin{array}{c}\text { SBL (standard } \\
\text { units), M } \pm \text { m }\end{array}$ & & & \\
\hline
\end{tabular}

Table 3: Distribution of the risk factors of bronchiolitis in RA patients with CT signs of this pathology.

\section{Discussion}

According to the chest CT report, our study found a comparatively high incidence of bronchiolitis $(29,5 \%)$ that was reported in the studies of other authors $[2,6-8,15,17]$.

The CT signs of small distal bronchioles involvement in combination bronchi can be one more evidence, that rheumatoid bronchiolitis is part of diffuse inflammatory-sclerotic involvement bronchial trees in RA [4-6,14].

In our results shown as low findings of clinical symptoms of rheumatoid bronchiolitis (only in $52.2 \%$ of RA patients with CT signs of bronchiolitis), on the one hand, are consistent with the literature data $[4,5]$, on the other hand, apparently, due to low sensitivity of conventional clinical methods for diagnosing this pathology. Therefore, it is unfair to talk about the uncommon of distal bronchial lesions in RA, it is correct to talk about the low 
and asymptomatic rheumatoid bronchiolitis based on the results of generally accepted clinical diagnostic methods, which nowadays requires the necessity to use $\mathrm{CT}$ of the lungs in the diagnosis of bronchioles involvement in patients with RA $[2,6,15]$.

The results of our work indicate a significant association of bronchiolitis development in RA with smoking, male patients, young age of RA onset, the presence of rheumatoid nodules, and high levels of DAS28, RF, ADCP, and SBL.

Similar results were reported by other authors: the presence of a connection between the development of rheumatoid bronchiolitis and the male sex of patients $[2,6,18,19]$, a high level of DAS28 $[2,6,16,17,21]$, young age of RA onset [2], smoking [2,20], high activity of RA [16], high levels of RF [17,21] and ACCP [2,11,21]. At the same time, in the work of some authors, no connection was found between the development of rheumatoid bronchiolitis with the presence of high levels of $\mathrm{RF}[2,19,22]$.

At the same time, for the first time in RA patients, we found a connection between the development of bronchiolitis and a high SBL level (Table 3).

However, according to our data, there is no connection between the development of bronchiolitis in RA with the age of patients and the duration of the disease. At the same time, in some studies, there is no relation between the development of rheumatoid bronchiolitis with the duration of RA $[2,17]$, while in other studies, conversely, the presence of this connection is noted [6]. Some works indicate the presence of a relation between the development of rheumatoid bronchiolitis and the age of patients with RA $[2,6,18]$.

Thus, our data indicate a relatively high frequency of detection of bronchiolitis in RA patients based on lung CT results, a low frequency of detection of clinical symptoms of rheumatoid bronchiolitis, and a relationship between the development of bronchiolitis in RA with smoking, male sex of patients, early age of RA onset, presence of rheumatoid knots and high levels of DAS28, RF, ACCP and SBL.

\section{Conclusion}

- $\quad$ Rheumatoid bronchiolitis according to the results of CT scan of the lungs is found in $29.5 \%$ of cases and is a relatively frequent extra-articular manifestation of RA.
- In patients with RA, clinical symptoms of bronchiolitis are found significantly less frequently than CT signs.

- $\quad$ Risk factors for the development of bronchiolitis in RA patients are smoking, male sex of patients, early age debuted RA, the presence of rheumatoid nodules, and high levels of DAS28, RF, ACCP, and SBL.

\section{Bibliography}

1. Turesson C. "Extra-articular rheumatoid arthritis". Current Opinion on Rheumatology 25.3 (2013): 360-366.

2. Nesterovich II., et al. "Complex clinical and nstrumental evaluation of lung injury in patients with rheumatoid arthritis". Nauchno-Prakticheskaya Revmatologiya = Rheumatology Science and Practice 54.5 (2006): 535-542.

3. Olson AL., et al. "Rheumatoid arthritis - interstitial lung disease-associated mortality". American Journal of Respiratory and Critical Care Medicine 183.3 (2011): 372-378.

4. Lahdensuo A., et al. "Bronchiolitis in rheumatoid arthritis". Chest 85 (1984): 705-798.

5. Schwarz MI., et al. "Bronchiolitis obliterans: the lone manifestation of rheumatoid arthritis". European Respiratory Journal 7 (1994): 817-820.

6. Sheyanov MV., et al. "Lesions of the distal bronchi in patients with rheumatoid arthritis". Scientific - Practical Rheumatology 6 (2009): 14-19.

7. Mori S., et al. "Small airway obstruction in patients with rheumatoid arthritis”. Modern Rheumatology 21.2 (2013): 164-173.

8. Avnon LS., et al. "Pulmonary functions testing in patients with rheumatoid arthritis". Israel Medical Association Journal (IMAJ) 11.2 (2009): 83-87.

9. Mamasaidov AT., et al. "Spontaneous antigen-specific B-cell activity in early rheumatoid arthritis and reactive arthritis". Scientific and practical rheumatology (Moscow) 51 (2013): 97.

10. Mamasaidov AT., et al. "The incidence and nature of the pathology of the respiratory system in rheumatoid arthritis". Central Asian Medical Journal 19 (2013): 59.

11. Aubart F., et al. "High levels of anti-cyclic citrullinated peptide autoantibodies are associated with co-occurrence of pulmonary diseases with rheumatoid arthritis". Journal of Rheumatology 38.6 (2011): 979-982. 
12. Mamasaidov AT., et al. "Indicators of spontaneous proliferation of B-lymphocytes in the diagnosis of neurological manifestations of rheumatoid arthritis". Journal of Neurology and Psychiatry 109.9 (2009): 63-66.

13. Brown KK. "Rheumatoid lung disease". Proceedings of the American Thoracic Society 4 (2007): 443-448.

14. Homma S., et al. "Diffuse panbronchiolitis in rheumatoid arthritis". European Respiratory Journal 12 (1998): 444-452.

15. Ternovoy SK., et al. "Multislice computed tomography in the diagnosis of lung lesions in patients with rheumatoid arthritis". Meditsinskaya Vizualizatsiya 5 (2009): 33-38.

16. Perez-Darame R., et al. "Rheumatoid arthritis-associated interstitial lung disease: lung inflammation evaluated with high resolution computed tomography scan is correlated to rheumatoid arthritis disease activity". Reumatology Clinic 11 (2015): 12-16.

17. Habib HM., et al. "Pulmonary involvement in early rheumatoid arthritis patients". Clinical Rheumatology 30.2 (2011): 217221.

18. Assayag D., et al. "Predictors of mortality in rheumatoid arthritis- related interstitial lung disease". Respirology 19.4 (2014): 493-500.

19. Kelly CA., et al. "Rheumatoid arthritis-related interstitial lung disease: associations, prognostic factors and physiological and radiological characteristics - a large multicenter UK study. Rheumatology (Oxford) 53.9 (2014): 1676-1682.

20. Gochuico BR., et al. "Progressive preclinical interstitial lung disease in rheumatoid arthritis". Archives of Internal Medicine 168.2 (2008): 159-166.

21. Bestaev DV., et al. "Comparative clinical, laboratory, and instrumental evaluation of interstitial lung changes in rheumatoid arthritis". Nauchno-Prakticheskaya Revmatologiya = Rheumatology Science and Practice 52.3 (2014): 277-282.

22. Yin Y., et al. "Anti-cyclic citrullinated peptide antibody is associated with interstitial lung disease in patients with rheumatoid arthritis. PLoS One 9.4 (2014): e92449.

\section{Volume 5 Issue 8 August 2021}

(C) All rights are reserved by Mamasaidov AT., et al. 